\title{
Analysis of turbulent Hydromagnetic flow with Radiative heat over a moving vertical plate in a Rotating System
}

\author{
Dawit H. Gebre' ${ }^{1}$ O. D. Makinde ${ }^{2}$, M. Kinyanjui ${ }^{3}$ \\ ${ }^{1}$ Pan African University Institute of Basic Sciences, Technology and Innovation Department of Computational Mathematics, Nairobi, \\ Kenya \\ ${ }^{2}$ Faculty of Military Science, Stellenbosch University, Private Bag x2, saldanha 7395, South Africa \\ ${ }^{3}$ Jomo Kenyatta University of Agriculture and Technology, Department of Pure and Applied Mathematics, Nairobi, Kenya
}

\section{Email address:}

davechrist36@yahoo.com (Dawit H. Gebre),makinded@gmail.com (O. D. Makinde), mathewkiny@yahoo.com (M. Kinyanjui)

\section{To cite this article:}

Dawit H. Gebre, O. D. Makinde, M. Kinyanjui . Analysis of Turbulent Hydromagnetic Flow with Radiative Heat over a Moving Vertical Plate in a Rotating System. Applied and Computational Mathematic. Vol. 3, No. 3, 2014, pp. 100-109.

doi: 10.11648/j.acm.20140303.15

\begin{abstract}
In this paper, the combined effects of magnetic fields, buoyancy force, thermal radiation, viscous and Ohmic heating on turbulent hydromagnetic flow of an incompressible electrically conducting fluid over a moving vertical plate in a rotating system is investigated numerically. The governing equations are reduced to non-linear ordinary differential equations using the time-averaged approach known as Reynolds-averaged Navier-Stokes equations (or RANS equations) and tackled by employing an efficient Runge-Kutta Fehlberg integration technique coupled with shooting scheme. Graphical results showing the effects of various thermophysical parameters on the velocity, temperature, local skin friction and local Nusselt number are presented and discussed quantitatively.
\end{abstract}

Keywords: Turbulent Flow, Magnetohydrodynamics (MHD), Radiative Heat, Time Averaging, Rotating System

\section{Introduction}

Turbulent flow is a flow regime characterized by chaotic variation in the fluid properties, such as low momentum diffusion, high momentum convection, and rapid variation of pressure and velocity in space and time. In turbulent flow, drag due to boundary layer increases and the unsteady vortices appear on many scales and interact with each other [1]. Turbulence causes the formation of eddies of many different length scales. Studies related to turbulent flow and heat transfer not only present a mathematical challenge but find several applications in many industrial, engineering and technological processes [2]. For instance, the external flow over all kind of vehicles such as cars, airplanes, ships and submarine are turbulence. The flow conditions in many industrial equipment such as pipes, ducts, precipitators, gas scrubbers, dynamic scraped surface heat exchangers, internal combustion engines and gas turbines are turbulence. In many geophysical flows such as rivers and atmospheric boundary layer, the flow turbulence is dominated by the coherent structure activities and associated turbulent events. In the medical field of cardiology, a stethoscope is used to detect heart_sounds and bruits, which are due to turbulent blood flow. Moreover, when flow is turbulent, particles exhibit additional transverse motion which enhances the rate of energy and momentum exchange between them thus increasing the heat transfer and the friction coefficient [3].

Meanwhile, hydromagnetic flow and heat transfer have received considerable attention in recent years due to its various applications in science, engineering and industries. Melt refining involves magnetic field applications to control excessive heat transfer rate. Other applications of magnetohydrodynamic (MHD) heat transfer include MHD generators, plasma propulsion in astronautics, nuclear reactor thermal dynamics and ionized-geothermal energy systems [4-6]. Model studies of the phenomena of MHD convection with respect to turbulent flow have been made by many authors. Burr et al. [7] presented the analysis of hydromagnetic turbulent flow and heat transfer in a rectangular duct with strong sidewall jets. Ji et al. [8] conducted a numerical investigation into turbulent pipe flow under the influence of an imposed transverse magnetic field. Kenjere and Hanjalic [9] numerically studied the effects of Lorentz force in turbulence closure models. Kitamura and Hirata [10] investigated the problem of turbulent heat and momentum transfer for electrically 
conducting fluid flowing in two-dimensional channel under the influence of an imposed transverse magnetic field. Numerical simulation of large-eddy in conductive flows at low magnetic Reynolds number was reported by Knaepen and Moin [11]. Kobayashi [12] also presented large eddy simulation of hydromagnetic turbulent channel flows with local subgrid-scale model based on coherent structures. Meanwhile, all conducting fluids with a temperature greater than absolute zero emit thermal radiation. Thermal radiation is the emission of electromagnetic waves. It represents a conversion of thermal energy into electromagnetic energy [13]. Thermal energy results in kinetic energy in the random movements of molecules in the conducting fluid. Therefore, whenever the temperature of surrounding fluid is high, the radiation effects play a very important role in the flow process [14]. This situation does occur in many engineering and industrial flow systems. In such cases one has to take into account the effects of radiation and free convection. Aboeldahab and Gendy [15] studied the radiation effects on MHD free convective flow of a gas past a semi-infinite vertical plate with variable thermophysical properties for higher-temperature difference. Ishak [16] investigated the thermal radiation effects on hydro-magnetic flow due to an exponentially stretching sheet. Furthermore, hydromagnetic flow in a rotating environment as received significant attention of several researchers due to its applications in various technological situations which are governed by the action of Coriolis force. Oceanography, meteorology, atmospheric science and limnology all contain some important and essential features of rotating fluids. An order of magnitude analysis shows that in basic field equations the effects of Coriolis force is more significant as compared to that of inertia and viscous forces. It is worthy to note that Coriolis and magnetohydrodynamic forces are comparable in magnitude and Coriolis force induces secondary flow in the fluid. Seth et al. [17] investigated unsteady hydromagnetic Couette flow of a viscous incompressible and electrically conducting fluid in a rotating system in the presence of uniform transverse magnetic field considering different aspects of the problem. Kinyanjui et al. [18] investigated the effect of hall current on MHD turbulent flow over a vertical plate in a rotating system. From the literature review, it appears that the combined effects of magnetic fields, buoyancy force, thermal radiation, viscous and Ohmic heating on turbulent hydromagnetic boundary layer flow in a rotating system has not been reported.

The main objective of this present study is to extend the theoretical model of MHD turbulent flow in a rotating system as described in [18] to include the combined effects of magnetic fields, buoyancy force, thermal radiation, viscous and Ohmic heating. The mathematical formulation of the problem based on the Reynolds averaged NavierStokes (or RANS) method is established and shooting method coupled with Runge-Kutta Fehlberg integration scheme is employed to tackle the problem in section two. Both numerical and graphical results are presented and discussed quantitatively with respect to various parameters embedded in the system in section three.

\section{Model Formulation}

Consider the hydromagnetic turbulent flow of an incompressible electrically conducting fluid past a moving vertical plate in a rotating system with thermal radiation in the presence of a uniform transverse magnetic field of strength $B_{0}$. Choose the coordinate system in such a way that $x$-axis is along the plate in upward direction, $z$ - axis normal to plane of the plate in the fluid and $y$ - axis perpendicular to $x z$ - plane. The uniform transverse magnetic field $\mathrm{B}_{0}$ is applied in a direction which is parallel to $z$ - axis. It is assumed that no applied or polarization voltages exist so electric field is zero. The magnetic Reynolds number is very small and the induced magnetic field produced by fluid motion is negligible in comparison to applied one. The plate moves along $\mathrm{x}$-axis with velocity $U_{0}$ and both the plate and the fluid rotate in unison with uniform angular velocity $\Omega$ about $z$ - axis as shown in figure 1 .

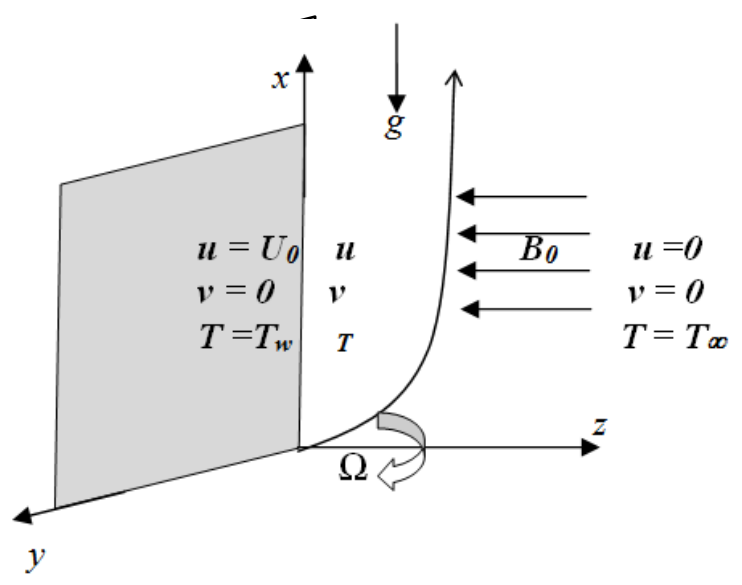

Figure 1. Geometry of the problem

Taking into consideration the assumptions made above together with the application of Reynolds averaged NavierStokes (or RANS) methods, the governing equations for hydromagnetic turbulent boundary layer flow of a viscous, incompressible, electrically conducting fluid with Hall effects and thermal radiations, under Boussinesq approximation, in a rotating frame of reference are given by $[10,14,18,20]$

$$
\begin{aligned}
& -2 \Omega \bar{v}=v \frac{d^{2} \bar{u}}{d z^{2}}+\frac{d}{d z}\left(n^{2} z^{2}\left(\frac{d \bar{u}}{d z}\right)^{2}\right)+g \beta\left(\bar{T}-T_{\infty}\right)- \\
& \frac{\sigma B_{0}^{2}}{\rho}\left(\frac{\bar{u}-m \bar{v}}{1+m^{2}}\right) \\
& 2 \Omega \bar{u}=v \frac{d^{2} \bar{v}}{d z^{2}}+\frac{d}{d z}\left(n^{2} z^{2}\left(\frac{d \bar{v}}{d z}\right)^{2}\right)-\frac{\sigma B_{0}^{2}}{\rho}\left(\frac{\bar{v}+m \bar{u}}{1+m^{2}}\right)
\end{aligned}
$$




$$
\begin{aligned}
& \alpha \frac{d^{2} \bar{T}}{d z^{2}}+\frac{d}{d z}\left(\frac{n^{2} z^{2}}{\operatorname{Pr}_{t}} \frac{d \bar{u}}{d z} \frac{d \bar{T}}{d z}\right)-\frac{1}{\rho c_{p}} \frac{d q_{r}}{d z}+ \\
& \frac{v}{c_{p}}\left[\left(\frac{d \bar{u}}{d z}\right)^{2}+\left(\frac{d \bar{v}}{d z}\right)^{2}\right]+\frac{\sigma B_{0}^{2}}{\rho c_{p}}\left[\frac{(\bar{u}-m \bar{v})^{2}+(\bar{v}+m \bar{u})^{2}}{\left(1+m^{2}\right)^{2}}\right]=0
\end{aligned}
$$

with

$$
\begin{aligned}
& \bar{u}=U_{0}, \bar{v}=0, \bar{T}=T_{w}, \text { at } \mathrm{z}=0, \\
& \bar{u}=0, \bar{v}=0, \bar{T}=T_{\infty}, \text { as } \mathrm{z} \rightarrow \infty
\end{aligned}
$$

heat at constant pressure, $\beta$ is the thermal expansion coefficient and $\operatorname{Pr}_{t}$ is the turbulence Prandtl number. It is assume that the fluid medium is optically thin with relatively low density and following Cogley et al.[13], the radiative heat flux $q_{r}$ is given as

$$
\frac{\partial q_{r}}{\partial z}=-4 \varepsilon^{2}\left(\bar{T}-T_{\infty}\right)
$$

where $\varepsilon<<1$ is the radiation absorption coefficient. Moreover, it is important to note that in order to obtain the turbulent flow model equations (1)-(3), we have decomposed the flow variables $u, v$ and $T$ into time-mean and fluctuating part, i.e.

$$
\begin{aligned}
& u=\bar{u}+u^{\prime}, \quad v=\bar{v}+v^{\prime}, \quad T=\bar{T}+T^{\prime}, \quad \bar{u}=\frac{1}{t} \int_{0}^{t} u d t, \\
& \bar{v}=\frac{1}{t} \int_{0}^{t} v d t, \quad \bar{T}=\frac{1}{t} \int_{0}^{t} T d t,
\end{aligned}
$$

and apply the time-averaging on time dependent momentum and energy balance equations under laminar flow scenario such that the time averaging of fluctuating part (with prime symbol) is zero. Both Boussinesq approximation and Prandtl mixing length hypothesis [20] are also applied to resolve the time-averaging of product of fluctuating part i.e.

$$
\begin{aligned}
& \frac{\partial \bar{u}}{\partial t}=\frac{\partial \bar{v}}{\partial t}=\frac{\partial \bar{T}}{\partial t}=0, \overline{u^{\prime} w^{\prime}}=-n^{2} z^{2}\left(\frac{\partial \bar{u}}{\partial z}\right)^{2}, \overline{v^{\prime} w^{\prime}}=-n^{2} z^{2}\left(\frac{\partial \bar{v}}{\partial z}\right)^{2}, \\
& \overline{T^{\prime} w^{\prime}}=-\varepsilon_{H} \frac{\partial \bar{T}}{\partial z}, \quad \varepsilon_{H}=\frac{\varepsilon_{m}}{\operatorname{Pr}_{t}}, \\
& \varepsilon_{m}=n^{2} z^{2} \frac{\partial \bar{u}}{\partial z}, \frac{\partial^{2}}{\partial x^{2}}<<\frac{\partial^{2}}{\partial z^{2}}, \frac{\partial \bar{u}^{\prime 2}}{\partial x}<<\frac{\partial \overline{u^{\prime} w^{\prime}}}{\partial z}, \frac{\partial \bar{v}^{\prime 2}}{\partial x}<<\frac{\partial \overline{v^{\prime} w^{\prime}}}{\partial z}, \\
& \frac{\partial \overline{u^{\prime} T^{\prime}}}{\partial x}<<\frac{\partial \overline{T^{\prime} w^{\prime}}}{\partial z},
\end{aligned}
$$

where the terms $\varepsilon_{M}$ and $\varepsilon_{H}$ are known as the momentum eddy diffusivity and the thermal eddy diffusivity. We introduce the following dimensionless variables and parameters into equations (1)-(5);

$$
\begin{aligned}
& \eta=\frac{z U_{0}}{v}, U=\frac{\bar{u}}{U_{0}}, V=\frac{\bar{v}}{U_{0}}, \theta=\frac{\bar{T}-T_{\infty}}{T_{w}-T_{\infty}}, M=\frac{\sigma B_{0}^{2} v}{\rho U_{0}^{2}}, m=w_{e} \tau_{e} \\
& R=\frac{\Omega v}{U_{0}^{2}}, \operatorname{Pr}=\frac{v}{\alpha}, E c=\frac{U_{0}^{2}}{c_{p}\left(T_{w}-T_{\infty}\right)}, G r=\frac{g \beta v\left(T_{w}-T_{\infty}\right)}{U_{0}^{3}} \\
& N r=\frac{4 \varepsilon^{2} v}{\rho c_{p} U_{0}^{2}}
\end{aligned}
$$

and obtain

$$
\begin{gathered}
-2 R V=\frac{d^{2} U}{d \eta^{2}}+\frac{d}{d \eta}\left(n^{2} \eta^{2}\left(\frac{d U}{d \eta}\right)^{2}\right)+G r \theta-M\left(\frac{U-m V}{1+m^{2}}\right) \\
2 R U=\frac{d^{2} V}{d \eta^{2}}+\frac{d}{d \eta}\left(n^{2} \eta^{2}\left(\frac{d V}{d \eta}\right)^{2}\right)-M\left(\frac{V+m U}{1+m^{2}}\right) \\
\frac{1}{\operatorname{Pr}} \frac{d^{2} \theta}{d \eta^{2}}+\frac{d}{d \eta}\left(\frac{n^{2} \eta^{2}}{\operatorname{Pr}_{t}} \frac{d U}{d \eta} \frac{d \theta}{d \eta}\right)+N r \theta+E c\left[\left(\frac{d U}{d \eta}\right)^{2}+\left(\frac{d V}{d \eta}\right)^{2}\right]+ \\
M E c\left[\frac{(U-m V)^{2}+(V+m U)^{2}}{\left(1+m^{2}\right)^{2}}\right]=0
\end{gathered}
$$

with

$$
\begin{aligned}
& U=1, \quad V=0, \quad \theta=1, \quad \text { at } \eta=0, \\
& U=0, V=0, \quad \theta=0, \text { as } \eta \rightarrow \infty,
\end{aligned}
$$

where $\mathrm{M}$ is the magnetic field parameter, $\mathrm{Nr}$ is the radiation parameter, $\mathrm{R}$ is the rotational parameter, Ec is the Eckert number, $\mathrm{Gr}$ is the Grashof number and Pr is the Prandtl number. Following Marchello and Toor [19], for high turbulence intensity, the turbulent Prandtl number is given in term of the fluid Prandtl number as $\operatorname{Pr}_{t}=\sqrt{\operatorname{Pr}}$. Other quantities of interest are the skin friction $\left(C_{f}\right)$ coefficient and the Nusselt number $(\mathrm{Nu})$ at the plate surface which are given as

$$
\begin{aligned}
& C_{f x}=\frac{\tau_{w x}}{\rho U_{0}^{2}}=-\left.\frac{d U}{d \eta}\right|_{\eta=0}, \quad C_{f y}=\frac{\tau_{w y}}{\rho U_{0}^{2}}=-\left.\frac{d V}{d \eta}\right|_{\eta=0}, \\
& N u=\frac{q_{w}}{k\left(T_{w}-T_{\infty}\right)}=-\left.\frac{d \theta}{d \eta}\right|_{\eta=0},
\end{aligned}
$$

where $\tau_{w x}=-\left.\mu \frac{d u}{d z}\right|_{z=0}, \quad \tau_{w y}=-\left.\mu \frac{d v}{d z}\right|_{z=0}$ and $\quad q_{w}=-\left.k \frac{d T}{d z}\right|_{z=0}$.

The nonlinear dimensionless model equations (10)-(13) together with the boundary conditions in (14)-(15) constitute a boundary value problem (BVP) and are solved numerically using shooting method coupled with RungeKutta Fehlberg integration scheme. This method involves, transforming equations (10)-(15) into a set of initial value problems (IVP) which contain unknown initial values that need to be determined by guessing, after which RungeKutta Fehlberg integration scheme is employed to integrate the set of initial valued problems until the given boundary conditions are satisfied. The entire computation procedure 
is implemented on computer using a program written in MAPLE language. From the process of numerical computation, the mean velocity and mean temperature are obtained. The numerical values are used to compute the skin friction coefficient and the Nusselt number as given in equation (15).

\section{Results and Discussion}

In order to understand the physical situation of the problem and effects of various parameters controlling the flow regime, we have computed the numerical values of mean velocities, mean temperature, the skin friction and the Nusselt number with respect to each parameter variation as shown in figures 2-31. It is important to note that $G r>0$ corresponds to cooling of the plate by convection current while $G r<0$ implies heating of the plate by convection current. Moreover, the cooling problem is often encountered in engineering applications such as cooling of electronic components and nuclear reactors. Numerical values in table 1 depict the effect of parameter variation on skin friction and Nusselt number. From the table, it is observed that the primary skin friction at the plate surface increases with an increase in parameter values of $M, R, N \mathrm{r}$, $\mathrm{Pr}, \mathrm{Gr}<0$ and decreases with an increase in parameter $m$, $E c$ and $G r>0$. The secondary skin friction increases with parameter $m, R, E c, G r>0$ and decreases with parameter $M$, $\mathrm{Nr}, \mathrm{Pr}, \mathrm{Gr}<0$. An increase in parameter $m, \mathrm{Nr}$ and $\mathrm{Pr}$ increases the Nusselt number while an increase in parameter values of $M, R, E c$ and $G r$ decreases the Nusselt number.

Table 1. Computation showing the skin friction and Nusselt number

\begin{tabular}{llllllllll}
\hline$M$ & $m$ & $R$ & $G r$ & $E c$ & $N r$ & $P r$ & $C_{f x}$ & $C_{f y}$ & $N u$ \\
\hline $\mathbf{1 . 0}$ & 0.1 & 1.0 & 0.1 & 0.1 & 1.0 & 0.71 & 1.21183514 & 0.81877576 & 0.83925868 \\
$\mathbf{2 . 0}$ & 0.1 & 1.0 & 0.1 & 0.1 & 1.0 & 0.71 & 1.51494067 & 0.70318128 & 0.82053306 \\
$\mathbf{3 . 0}$ & 0.1 & 1.0 & 0.1 & 0.1 & 1.0 & 0.71 & 1.78458544 & 0.63077322 & 0.80364695 \\
$\mathbf{1 . 0}$ & 0.5 & 1.0 & 0.1 & 0.1 & 1.0 & 0.71 & 1.21244422 & 0.94064112 & 0.84088432 \\
$\mathbf{1 . 0}$ & 1.0 & 1.0 & 0.1 & 0.1 & 1.0 & 0.71 & 1.14983533 & 1.02958701 & 0.84583895 \\
$\mathbf{1 . 0}$ & 0.1 & 2.0 & 0.1 & 0.1 & 1.0 & 0.71 & 1.55777192 & 1.28916619 & 0.82453955 \\
$\mathbf{1 . 0}$ & 0.1 & 3.0 & 0.1 & 0.1 & 1.0 & 0.71 & 1.84502594 & 1.62487996 & 0.80871950 \\
$\mathbf{1 . 0}$ & 0.1 & 1.0 & 0.5 & 0.1 & 1.0 & 0.71 & 1.04984232 & 0.86729541 & 0.83701858 \\
$\mathbf{1 . 0}$ & 0.1 & 1.0 & 1.0 & 0.1 & 1.0 & 0.71 & 0.84543532 & 0.92903706 & 0.83237814 \\
$\mathbf{1 . 0}$ & 0.1 & 1.0 & 0.5 & 0.1 & 1.0 & 0.71 & 1.45275334 & 0.74715631 & 0.84034948 \\
$\mathbf{1 . 0}$ & 0.1 & 1.0 & 1.0 & 0.1 & 1.0 & 0.71 & 1.65199584 & 0.68831179 & 0.83930426 \\
$\mathbf{1 . 0}$ & 0.1 & 1.0 & 0.1 & 0.5 & 1.0 & 0.71 & 1.20895408 & 0.82012949 & 0.52261924 \\
$\mathbf{1 . 0}$ & 0.1 & 1.0 & 0.1 & 1.0 & 1.0 & 0.71 & 1.20534494 & 0.82182793 & 0.12720102 \\
$\mathbf{1 . 0}$ & 0.1 & 1.0 & 0.1 & 0.1 & 2.0 & 0.71 & 1.21843079 & 0.81542480 & 1.41337547 \\
$\mathbf{1 . 0}$ & 0.1 & 1.0 & 0.1 & 0.1 & 3.0 & 0.71 & 1.22393362 & 0.81296122 & 2.06881414 \\
$\mathbf{1 . 0}$ & 0.1 & 1.0 & 0.1 & 0.1 & 1.0 & 4.00 & 1.23139532 & 0.81022002 & 3.49966374 \\
$\mathbf{1 . 0}$ & 0.1 & 1.0 & 0.1 & 0.1 & 1.0 & 7.10 & 1.23772125 & 0.80862109 & 6.20529525 \\
\hline & & & & & & & & & \\
\hline
\end{tabular}

\subsection{Effects of Parameter Variation on Mean Velocities}

Figures 2-8 illustrate the effects of parameter variation on the primary mean velocity component. Generally the primary mean velocity is highest at the moving plate surface and tends zero free stream value far away from the place surface satisfying the prescribed boundary conditions. An increase in the fluid rotation, magnetic field intensity, hall current, thermal radiation and Prandtl number decreases the momentum boundary layer thickness as shown in figures 2-6, consequently the primary mean velocity decreases. In figure 7 , it is observed that the momentum boundary layer thickness increases with cooling of the plate by convectional current $(\mathrm{Gr}>0)$ due to buoyancy force and decreases with heating of the plate by convectional current $(\mathrm{Gr}<0)$. As the viscous heating increases $(E c)$, the primary mean velocity increases leading to a rise in momentum boundary layer thickness as shown in figure 8. Figures 9-15 depict the secondary mean velocity for some selected values of the parameters. Interestingly, flow reversal is generally observed with its peak value within the boundary layer regime and zero secondary mean velocity at both the plate surface and free stream satisfying the prescribed boundary conditions. As the values of parameter $R, N r, M$ and $P r$ increase due to combined effects of fluid rotation, magnetic field and thermal radiation, the fluid flows toward the plate surface with a decrease in the reverse flow intensity as shown in figures 9-13. This is expected, since increase in magnetic field enhances the effect of Lorentz force which acts as a resistance to the flow, leading to a decrease in the momentum boundary layer thickness. Figure 14 shows that the secondary mean flow reversal and its peak values increase with cooling $(\mathrm{Gr}>0)$ and decreases with heating $(\mathrm{Gr}<0)$ of the plate surface by convectional current due to buoyancy force. Increase viscous heating (Ec) increases the secondary mean flow reversal and the momentum boundary layer thickness as shown figure 15 .

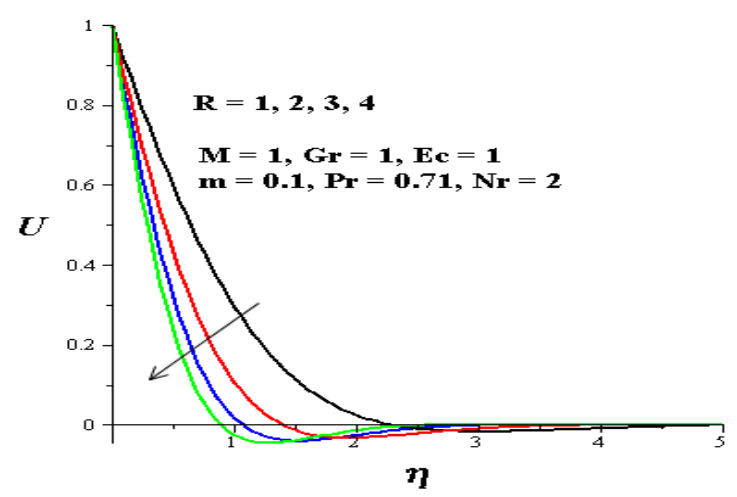

Figure 2. Primary velocity profiles with increasing $R$. 


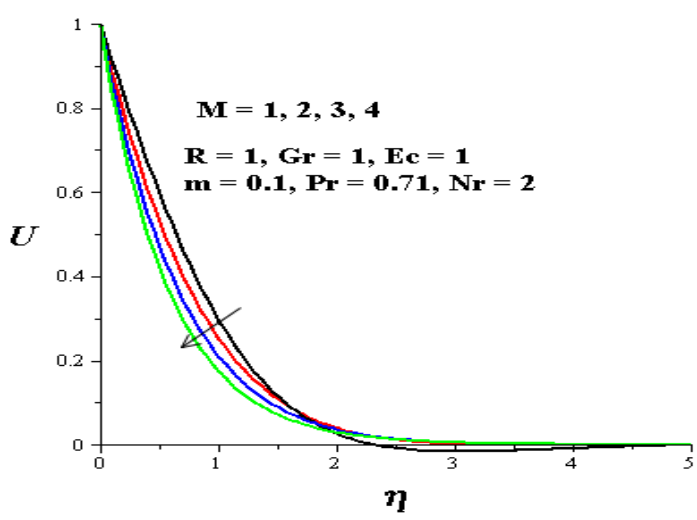

Figure 3. Primary velocity profiles with increasing $M$.

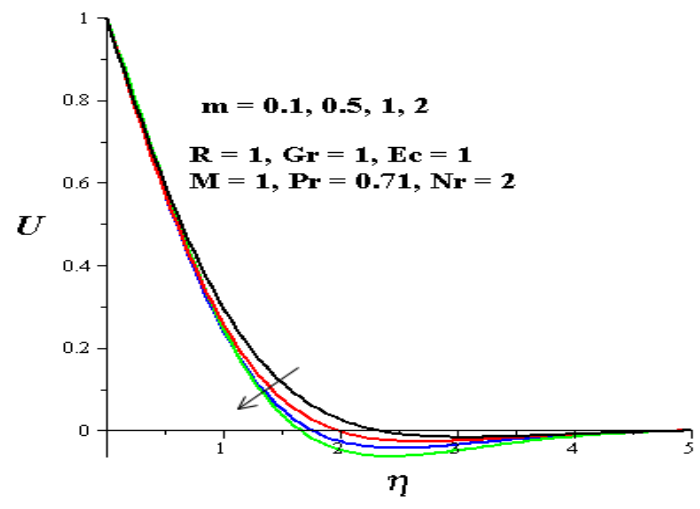

Figure 4. Primary velocity profiles with increasing $m$.

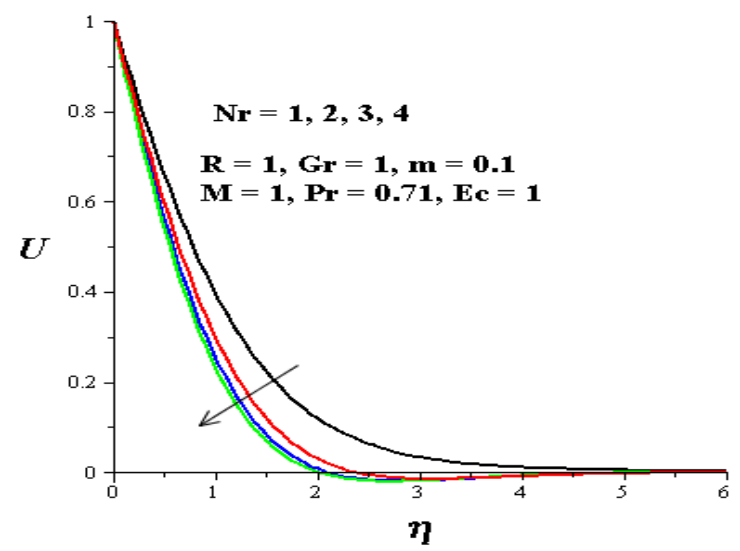

Figure 5. Primary velocity profiles with increasing $\mathrm{Nr}$.

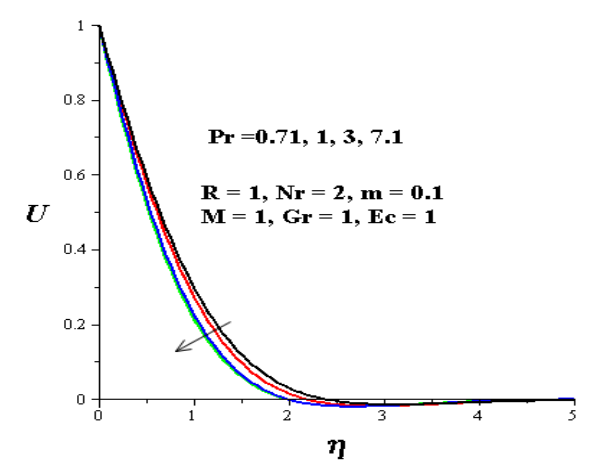

Figure 6. Primary velocity profiles with increasing Pr.

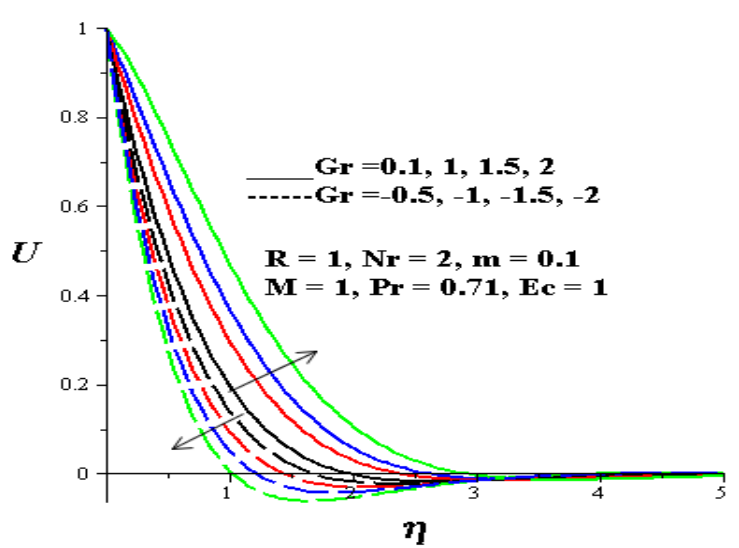

Figure 7. Primary velocity profiles with increasing $G r$.

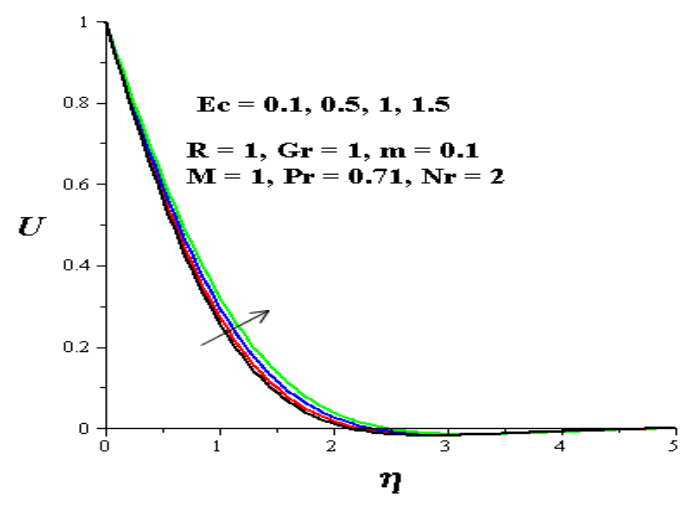

Figure 8. Primary velocity profiles with increasing Ec.

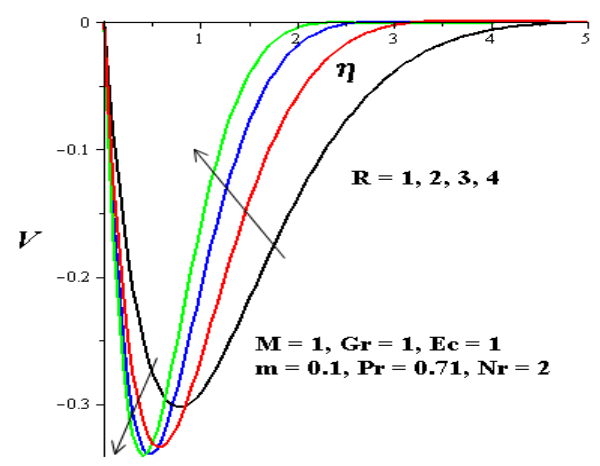

Figure 9. Secondary velocity profiles with increasing $R$.

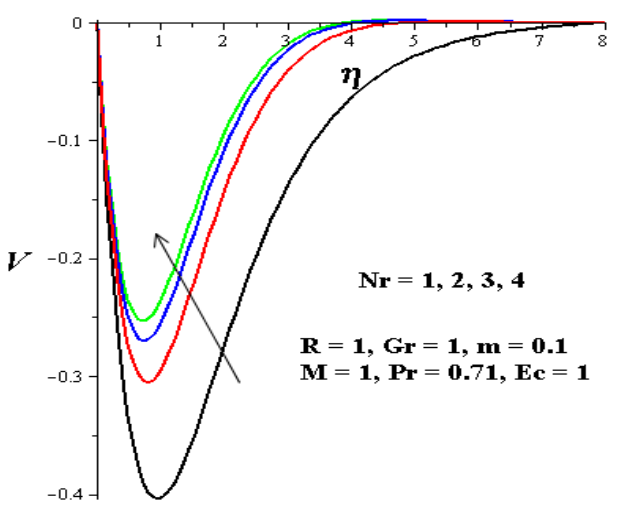

Figure 10. Secondary velocity profiles with increasing $\mathrm{Nr}$. 


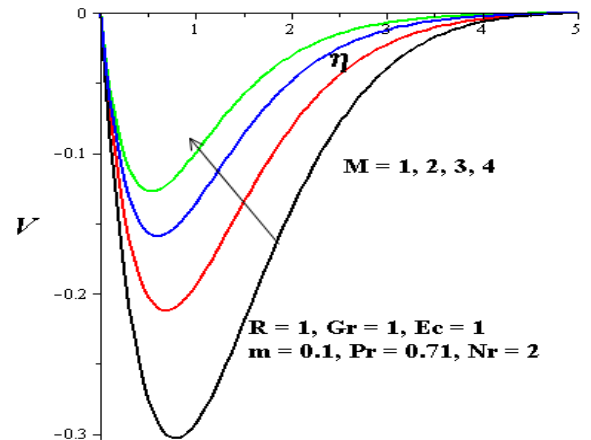

Figure 11. Secondary velocity profiles with increasing $M$.

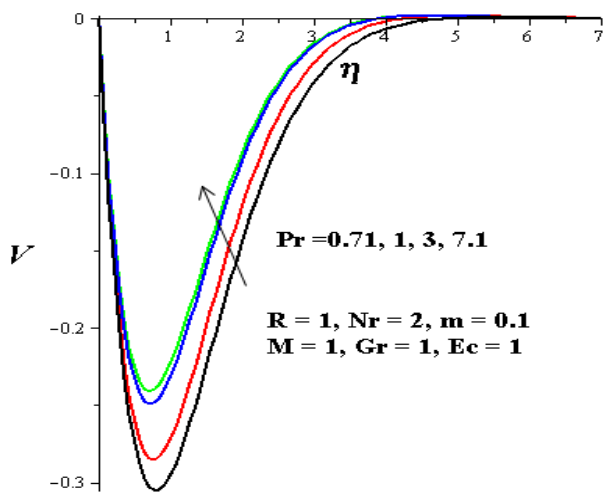

Figure 12. Secondary velocity profiles with increasing Pr.

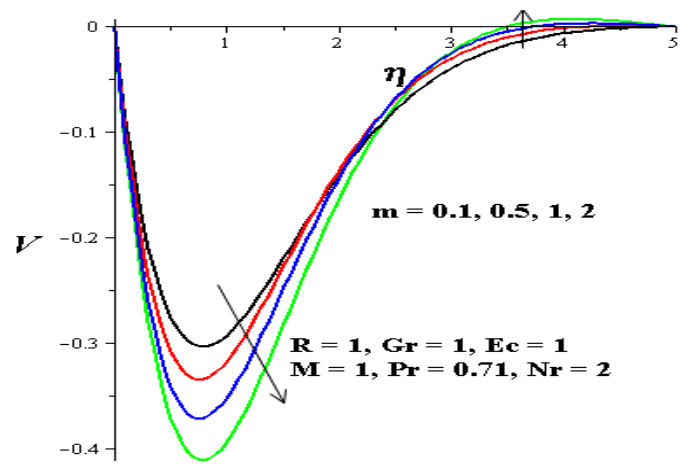

Figure 13. Secondary velocity profiles with increasing $m$.

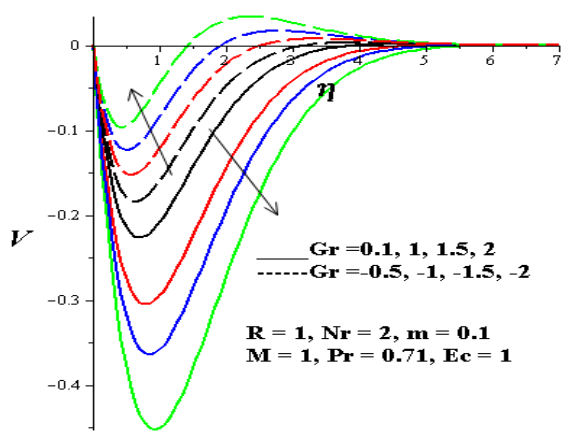

Figure 14. Secondary velocity profiles with increasing $G r$.

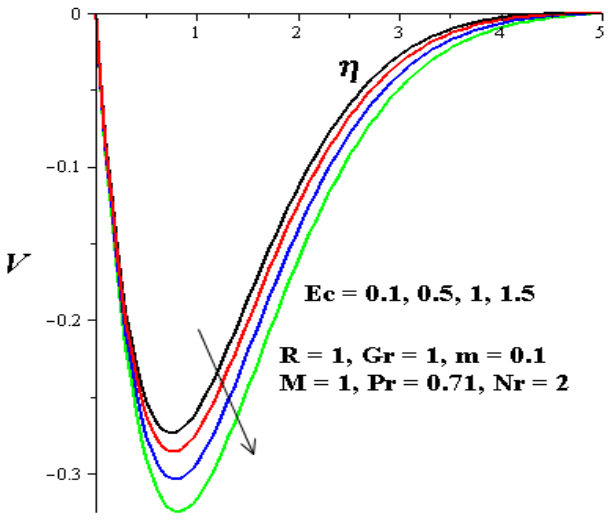

Figure 15. Secondary velocity profiles with increasing Ec.

\subsection{Effects of Parameter Variation on Mean Temperature Profiles}

Figures 16-22 illustrate the effects of various thermophysical parameters on the mean temperature profiles. It is noteworthy that the mean temperature decreases from the plate surface to the prescribed free stream zero value far away the plate. Meanwhile, as the parameter values of $R, M$ and $E c$ increase, the thermal boundary layer thickness increases near the plate surface as shown in figures 16-18. This may be attributed to the combined effects of fluid rotation, viscous and Ohmic heating, and leading to a rise in the mean temperature. In figures 19-21, a decrease in the thermal boundary layer thickness in observed with increasing parameter values of $N r, m$ and $P r$, consequently, the mean temperature decreases. Figure 22 shows that the mean temperature within the boundary layer regime is enhanced by plate cooling while the heating of the plate by buoyancy force decreases the boundary layer thickness. This can be explained by the fact that the heat is transferred from the plate to the fluid by buoyancy force during cooling leading to a rise in the mean temperature

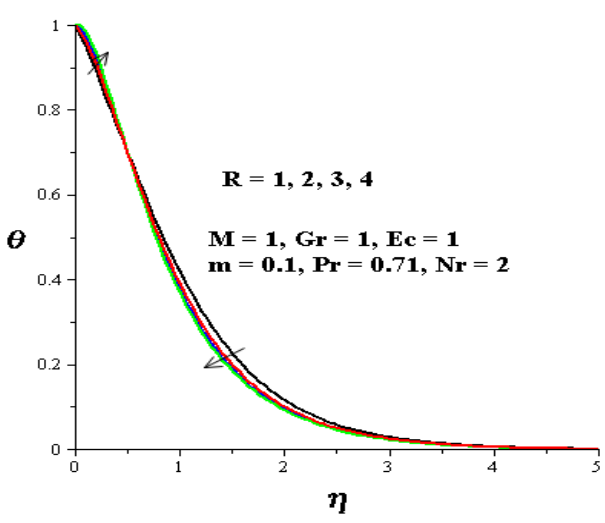

Figure 16. Temperature profiles with increasing $R$. 


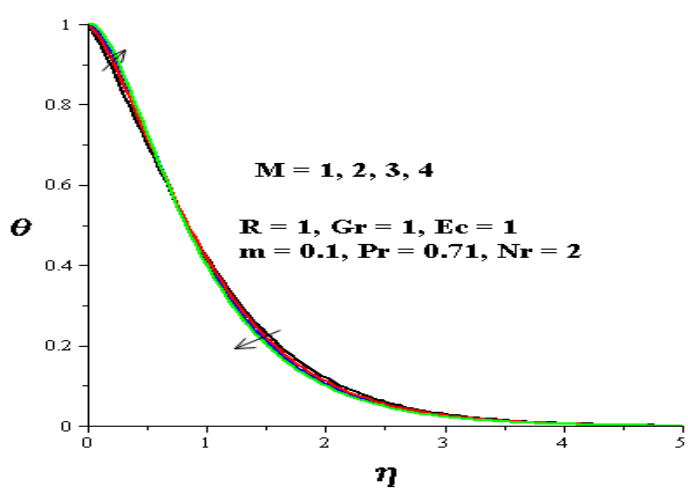

Figure 17. Temperature profiles with increasing $M$.

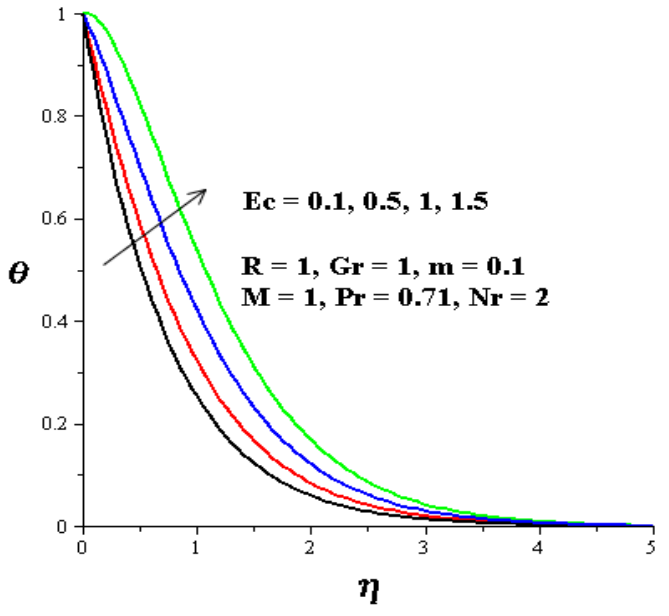

Figure 18. Temperature profiles with increasing Ec.

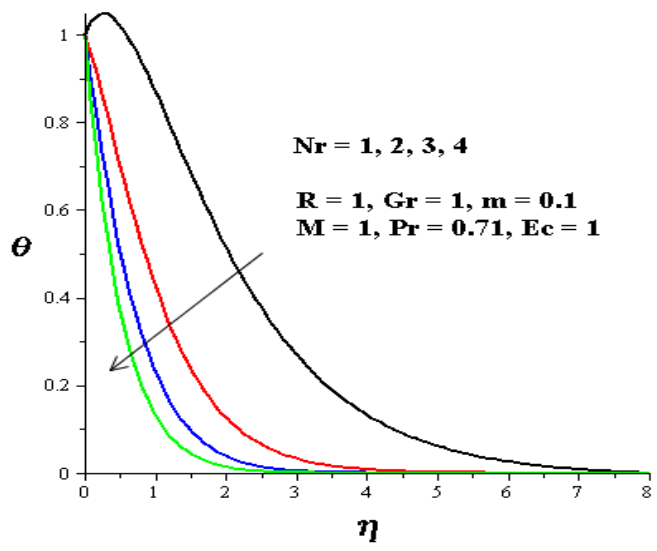

Figure 19. Temperature profiles with increasing Ec.

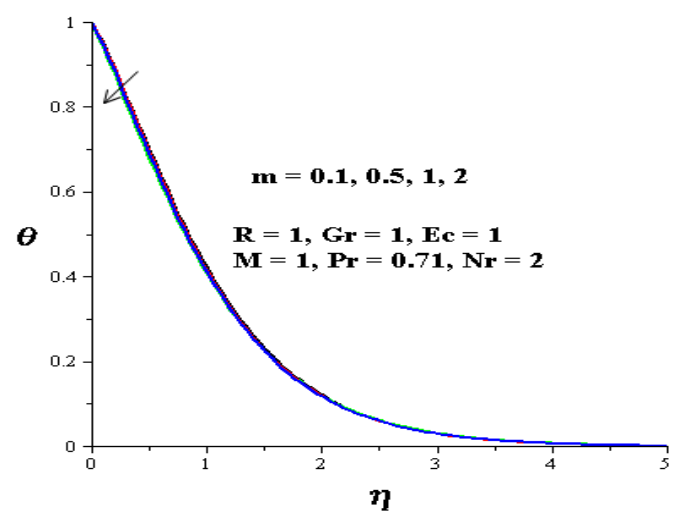

Figure 20. Temperature profiles with increasing $m$.

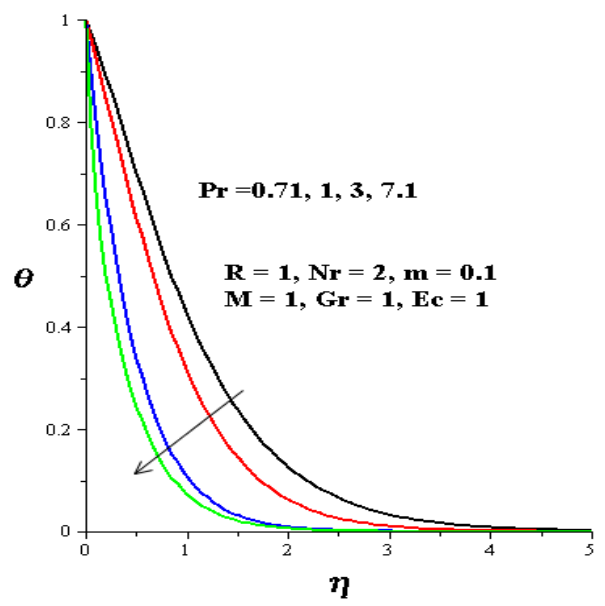

Figure 21. Temperature profiles with increasing Pr.

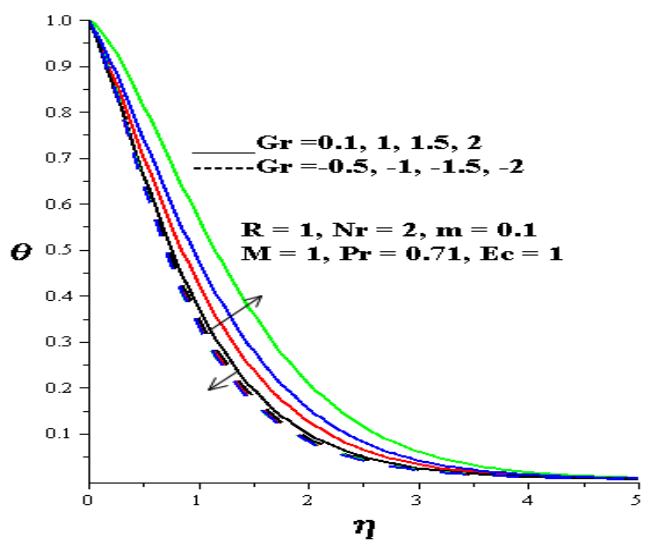

Figure 22. Temperature profiles with increasing $G r$.

\subsection{Effects of Parameter Variation on Skin Friction and Nusselt Number}

Figures 23-25 demonstrate the effects of increasing each thermophysical parameter on the primary skin friction. We observed that the primary skin friction increases with an increase in magnetic field intensity, fluid rotation, thermal radiation, Prandtl number and plate heating by convectional current. This can be attributed to a rise in mean velocity gradient at the plate surface. Meanwhile a fall in the primary skin friction is noticed with increasing intensity of 
Hall current, viscous heating and plate cooling due to buoyancy force. Figures 26-28 depict the effects of parameter variation on secondary skin friction. A combined increase in the fluid rotation, Hall current, viscous heating and plate cooling due to buoyancy force increases the secondary skin friction while an increase in the Ohmic heating, thermal radiation and Prandtl number decreases the secondary skin friction at the plate surface. In figures 29-31, the variation in Nusselt number is illustrated with different parameters. An increase in Hall current, thermal radiation and Prandtl number enhances the Nusselt number due to a rise in the temperature gradient at the plate surface. However, the Nusselt number decreases with a combined increase in Ohmic and viscous heating, fluid rotation and buoyancy force.

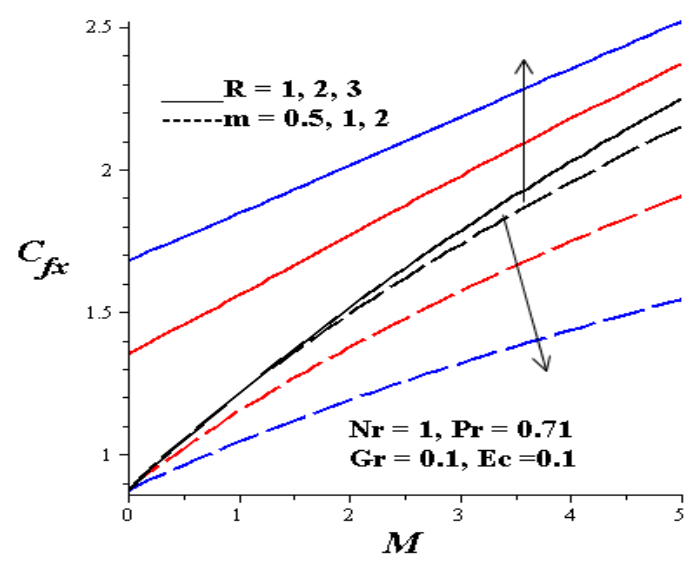

Figure 23. Primary skin friction with increasing $M, R$ and $m$.

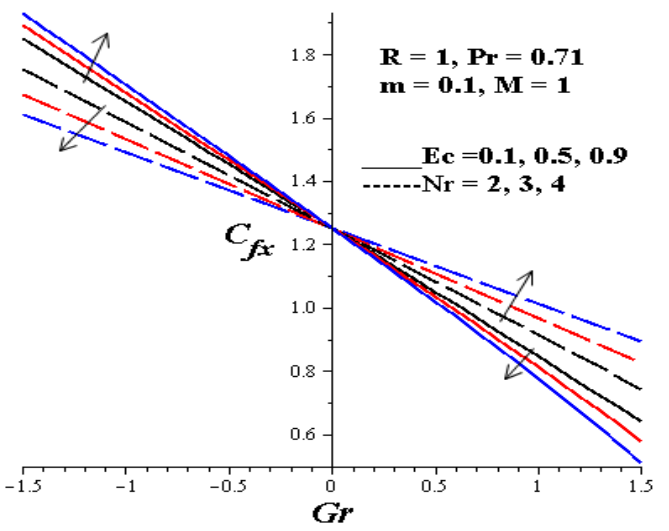

Figure 24. Primary skin friction with increasing $\mathrm{Ec}, \mathrm{Nr}$ and $\mathrm{Gr}$.

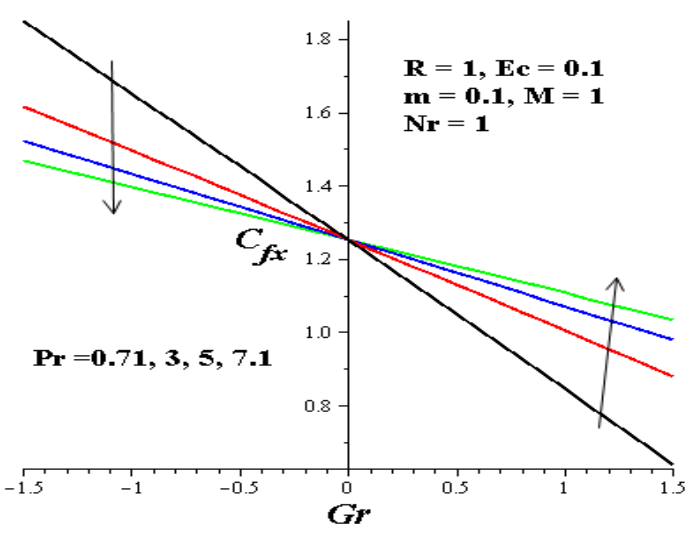

Figure 25. Primary skin friction with increasing $P r$.

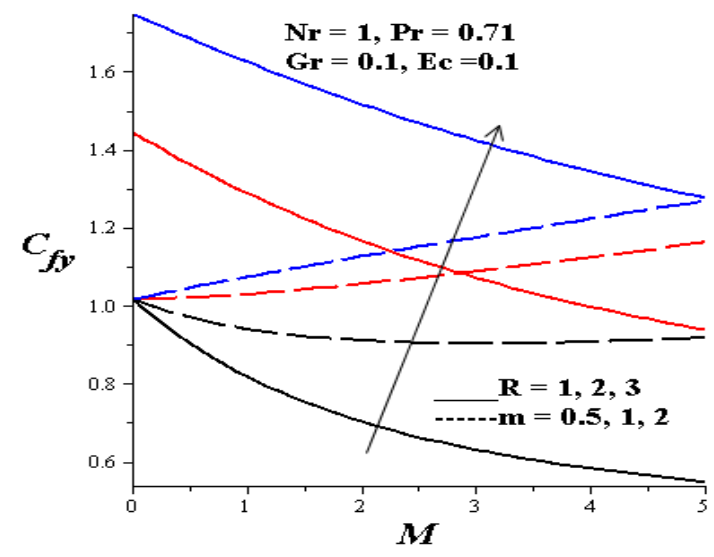

Figure 26. Secondary skin friction with increasing $M, R$ and $m$.

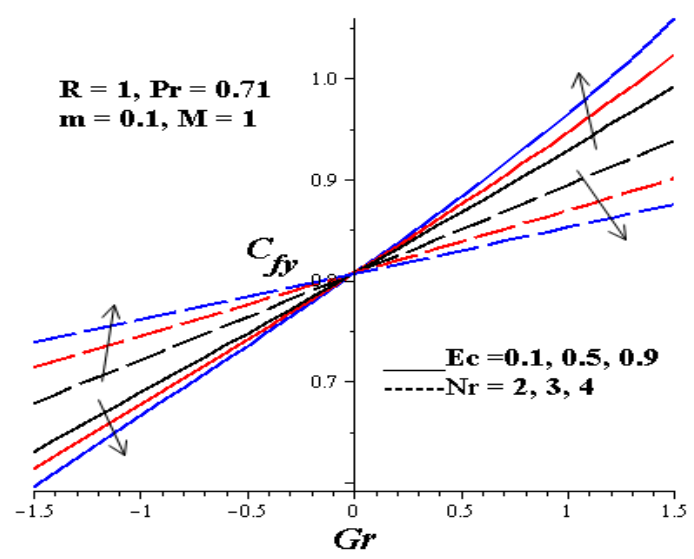

Figure 27. Secondary skin friction with increasing $\mathrm{Ec}, \mathrm{Nr}$ and $\mathrm{Gr}$. 


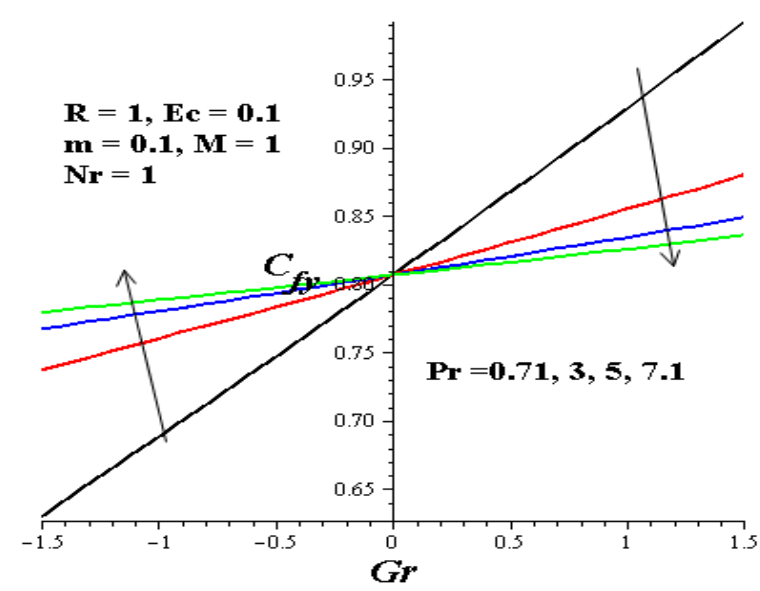

Figure 28. Secondary skin friction with increasing Pr.

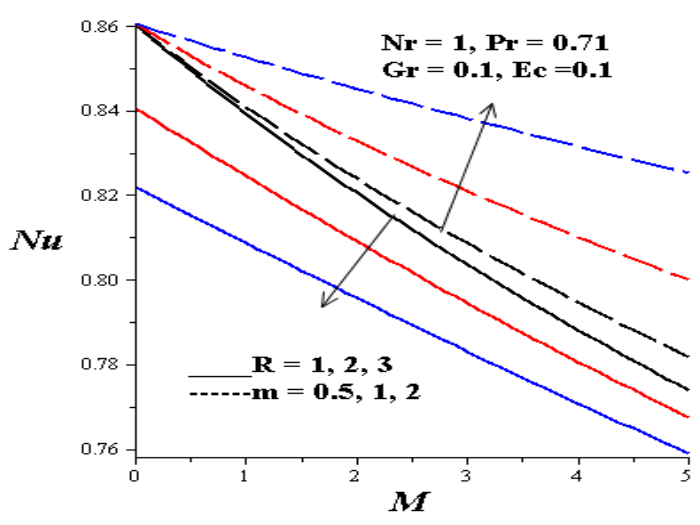

Figure 29. Nusselt number with increasing $M, R$ and $m$.

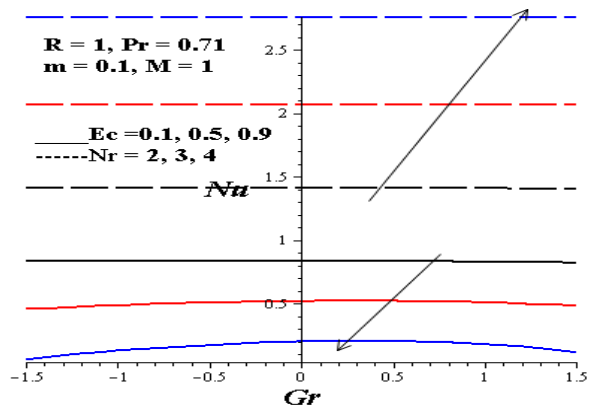

Figure 30. Nusselt number with increasing Ec, Nr and Gr.

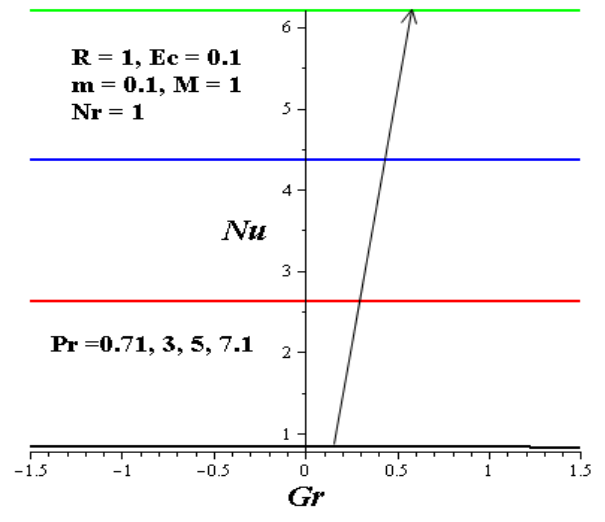

Figure 31. Nusselt number with increasing Pr.

\section{Conclusions}

The hydromagnetic turbulent flow of a conducting fluid over a moving plate in a rotating system with thermal radiation, viscous and Ohmic heating is numerically investigated. The effects of various thermophysical parameters on the mean velocity, mean temperature, skin friction and Nusselt number were obtained. Our results can be summarized as follows:

- The primary mean velocity and momentum boundary layer increases with $G r>0, E c$ and decreases with $R, M, m, N r, P r, G r<0$.

- The secondary mean velocity shows flow reversal and momentum boundary layer increases with $\mathrm{m}$, $\mathrm{Gr}>0, E c$ and decreases with $R, \mathrm{Nr}, \mathrm{M}, \mathrm{Pr}, \mathrm{Gr}<0$.

- The mean temperature and thermal boundary layer increases with $R, M, E c, G r>0$ and decreases with $\mathrm{Nr}, \mathrm{Pr}, \mathrm{m}, \mathrm{Gr}<0$.

- $\quad$ The primary skin friction increases with $M, R, N \mathrm{r}$, $P r, G r<0$ and decreases with $m, E c$ and $G r>0$.

- The secondary skin friction increases $m, R, E c$, $G r>0$ and decreases $M, N r, P r, G r<0$.

- The Nusselt number increase with $m, N r, P r$ increases and decreases with $M, R, E c, G r$.

\section{Acknowledgments}

The author would like to thank the Pan African University -African Union Commission for the financial support under its program of PAUISTI

\section{References}

[1] Narasimha R., Rudra Kumar S., Prabhu A., Kailas S.V. (2007). Turbulent flux events in a nearly neutral atmospheric boundary layer. Philosophical Transactions of the Royal Society A: Mathematical, Physical and Engineering Sciences (Phil Trans R Soc Ser A, Vol. 365, pp. 841-858) 365 (1852): 841-858.

[2] Trevethan M, Chanson H (2010). Turbulence and turbulent flux events in a small estuary. Environmental Fluid Mechanics, Vol. 10, pp. 345-368) 10 (3): 345-368

[3] Avila K., Moxey D., de Lozar A., Avila M., Barkley D., Hof B.( 2011). The onset of turbulence in pipe flow. Science 333 (6039): 192-196.

[4] Makinde O. D., Khan W. A., Khan Z. H. (2013). Buoyancy effects on MHD stagnation point flow and heat transfer of a nanofluid past a convectively heated stretching/shrinking sheet. International Journal of Heat and Mass Transfer 62, 526-533.

[5] Seini Y. I., Makinde O. D. (2013). MHD boundary layer due to exponentially stretching surface with radiation and chemical reaction. Mathematical Problems in Engineering, Volume 2013, 163614(7 pages).

[6] Nandkeolyar R., Seth G. S., Makinde O. D., Sibanda P., Ansari M. S. (2013). Unsteady hydromagnetic natural convection flow of a dusty fluid past an impulsively moving 
vertical plate with ramped temperature in the presence of thermal radiation. ASME-Journal of Applied MechanicsVol. 80, 061003(1-9).

[7] Burr, U., Barleon, L., Muller, U., Tsinober, A. (2000) Turbulent transport of momentum and heat in magnetohydrodynamic rectangular duct flow with strong sidewall jets. J. Fluid Mech. 406, 247-279.

[8] Ji, H.-C., Gardner, R. A. (1997). Numerical analysis of turbulent pipe flow in a transverse magnetic field. Int. J. Heat Mass Trans., 40, 1839-1851.

[9] Kenjeres, S., Hanjalic, K. (2000). On the implementation of effects of Lorentz force in turbulence closure models. Int. J. Heat Fluid Flow, 21, 329-337.

[10] Kitamura, K., Hirata, M. (1978). Turbulent heat and momentum transfer for electrically conducting fluid owing in two-dimensional channel under transverse magnetic field. Proc. 6th Int. Heat Transfer Conf., Toronto, Canada, 3, 159.

[11] Knaepen, B., Moin, P. (2004). Large-eddy simulation of conductive flows at low magnetic Reynolds number. Phys. Fluids, 16, 1255-1261.

[12] Kobayashi, H. (2006). Large eddy simulation of magnetohydrodynamic turbulent channel flows with local subgrid-scale model based on coherent structures. Phys. Fluids, 18, 045107.

[13] Cogley A. C., Vincent W. G., Giles S. E. (1968) Differential approximation to radiative heat transfer in a non-grey gas near equilibrium. AIAA J, 6:551-553.
[14] Makinde O. D., Tshehla M. S. (2014). Unsteady hydromagnetic flow of radiating fluid past a convectively heated vertical plate with the Navier slip. Advances in Mathematical Physics, Volume 2014, Article ID 973593, 10 pages.

[15] Aboeldahab E. M., EI Gendy M. S. (2002), Radiation effect on MHD free convective flow of a gas past a semi-infinite vertical plate with variable thermo physical properties for high-temperature difference, Can. J. Phys., 80, 1609-1619.

[16] Ishak A. (2011), MHD boundary layer flow due to an exponentially stretching sheet with radiation effect, Sains Malaysiana, 40, 391-395.

[17] Seth G.S., Nadkeolyar R., Ansari M. S (2012). Effects of Hall current and Rotation on Unsteady MHD couette flow in the presence of an Inclined Magnetic field. Journal of Applied Fluid Mechanics 5: 67-74.

[18] Kinyanjui M., Emmah M., Jackson K. (2012). Hydromagnetic turbulence flow of rotating system past a semi-infinite vertical plate with hall current. International Journal of pure and Applied mathematics Vol. 79, No. $197-$ 119.

[19] Marchello J. M., Toor H. L. (1963). A mixing model for transfer near a boundary. Ind. Eng. Chem. Fund., 2, 1, 8.

[20] Bejan, A. (1995). Convection Heat Transfer. Second Edition. John Wiley \& Sons Inc.: New York, New York. 